protein 1 (CHI3L1, also known as YKL-40) were validated with ELISA. Further, pirfenidone decreased the secretion of both DKK1 $(p=0.006)$ and OPG $(p=0.02)$ by SpA FLSs stimulated with TGF $\beta$, TNF $\alpha$, and INF $\gamma$, while the concentration of RANKL was below the detection limit of the ELISA assay in all cultures. Finally, pirfenidone inhibited the deposition of hydroxyapatite by osteoblasts in a dose-dependent manner $(p=0.0001)$. This inhibition was partly reversible when removing pirfenidone after the first week of the mineralization assay.

Conclusions: Taken together, pirfenidone inhibited SpA myofibroblast formation and activity and osteoblast mineralization. This encourages further research in anti-fibrotics as treatment of new bone formation in SpA.

Disclosure of Interest: None declared

DOI: 10.1136/annrheumdis-2017-eular.1503

\section{AB0120 ACCELERATED OSTEOGENIC DIFFERENTIATION OF HUMAN BONE-DERIVED CELLS IN ANKYLOSING SPONDYLITIS}

Y.-S. Park ${ }^{1}$, S. Jo ${ }^{2}$, I.-H. Sung ${ }^{3}$, T.-H. Kim ${ }^{2}{ }^{1}{ }^{1}$ Orthopaedic Surgery, Hanyang University Hospital, Guri; ${ }^{2}$ Rheumatology, Hanyang University Hospital for Rheumatic Disease: ${ }^{3}$ Orthopaedic Surgery, Hanyang University Hospital, Seoul, Korea, Republic Of

Background: Ankylosing spondylitis (AS) is characterized by excessive bone formation with syndesmophytes, leading to bony ankylosis. The contribution of osteoblasts to the pathogenesis of ankylosis is poorly understood.

Objectives: The aim of this study was to determine molecular differences between disease controls $(\mathrm{Ct})$ and $\mathrm{AS}$ bone-derived cells (BdCs) during osteogenic differentiation.

Methods: We confirmed osteoblastic differentiation of $\mathrm{Ct}$ and $\mathrm{AS}$ BdCs under osteogenic medium by observing morphological changes and measuring osteoblastic differentiation markers. Osteoblast differentiation was detected by alkaline phosphatase (ALP) staining and activity, and alizarin red $S$ and hydroxyapatite staining. Osteoblast-specific markers were analyzed by qRT-PCR, immunoblotting, and immunostaining. To examine the effects of inflammation, we added $\mathrm{AS}$ and healthy control serum to $\mathrm{Ct}$ and $\mathrm{AS} \mathrm{BdCs}$, and then analyzed osteoblast-specific markers.

Results: AS BdCs showed elevated basal intercellular and extracellular ALP activity compared to $\mathrm{Ct}$. When osteoblast differentiation was induced, AS BdCs exhibited higher expression of osteoblast-specific marker genes and faster mineralization than $\mathrm{Ct} \mathrm{BdCs}$, indicating that these cells differentiated more rapidly into osteoblasts. ALP activity and mineralization accelerated when serum from AS patients was added to $\mathrm{Ct}$ and AS BdCs.

Conclusions: Our results revealed that AS BdCs showed significantly increased osteoblastic activity and differentiation capacity by regulating osteoblast-specific transcription factors and proteins compared to $\mathrm{Ct}$ BdCs. Active inflammation caused by adding AS serum accelerated bony ankylosis. Our study could provide useful basic data for understanding the molecular mechanism of ankyloses in AS.

Disclosure of Interest: None declared

DOI: 10.1136/annrheumdis-2017-eular.4885

\section{SLE, Sjögren's and APS - etiology, pathogenesis and animal models}

\section{AB0121 DYSREGULATED CIRCULATING MIRNA LEVELS ARE CHARACTERISTIC OF BOTH NON SJÖGREN'S SICCA AND PRIMARY SJÖGREN'S SYNDROME PATIENTS}

A.P. Lopes $^{1,2}$, M.R. Hillen ${ }^{1,2}$, E. Chouri ${ }^{1,2}$, S.L. Blokland ${ }^{1,2}$, A.A. Kruize ${ }^{1}$, M. Rossato ${ }^{1,2}$, T.R. Radstake ${ }^{1,2}$, J.A. van Roon ${ }^{1,2} .{ }^{1}$ Department of Rheumatology \& Clinical Immunology; ${ }^{2}$ Laboratory of Translational Immunology, University Medical Centre Utrecht, Utrecht, Netherlands

Background: MicroRNAs are small non-coding RNAs that play important regulatory roles in a variety of biological processes. They can regulate the post-transcriptional expression of target genes and play an important role in gene regulation. Specific microRNAs are stably present in serum and changes in their abundance are potentially disease-specific. Considering their important role in regulation of the immune system, we investigated circulating levels of miRNAs in patients with primary Sjögren's syndrome (pSS) and those with non-Sjögren's sicca (nSS) in relation to disease activity.

Objectives: To assess the expression of a large number of miRNAs in the serum of pSS and nSS patients as compared to healthy controls and to investigate their correlation with disease activity.

Methods: Two independent cohorts (discovery and validation) were established, consisting of a total of $37 \mathrm{pSS}$ patients classified according to the 2002 criteria, 20 nSS patients that were not clinically considered to be pSS and did not meet the classification criteria, and 18 healthy controls $(\mathrm{HC})$. Serum miRNAs were isolated and miRNA profiling of 758 miRNA was performed using the OpenArray platform in the discovery cohort. A selection of 10 miRNAs found to be differentially expressed between the groups was measured in the independent validation cohort using single TaqMan microRNA Assays.

Results: miRNA profiling revealed 10 miRNAs to be differentially expressed between the groups; 2 in pSS vs HC, 7 in nSS vs $\mathrm{HC}$ and 1 in both pSS and nSS vs HC. One miRNA was excluded from further analysis after technical validation by single TaqMan microRNA Assay. The other 9 miRNAs were measured in the validation cohort. Surprisingly, 2 miRNAs were validated to be increased in the nSS group as compared to HC (snRNA-U6 and miR-661). Using the data from both cohorts combined, the levels of snRNA-U6 and miR-661 was associated with serum Ig and C4 in the nSS group, but also in the pSS group. This prompted us to investigate miRNA expression in subgroups of pSS patients. snRNA-U6 and miR-661 levels are significantly increased compared to $\mathrm{HC}$ in pSS patients negative for autoantibodies. In autoantibody positive pSS patients, levels of snRNA-U6 and miR-661 are comparable to those found in $\mathrm{HC}$ and both miRNAs are significantly increased in autoantibody negative patients as compared to autoantibody positive pSS patients. In addition, their expression is strongly associated with leukocyte numbers in the autoantibody positive patients, but not in the negative patients.

Conclusions: Increased circulating levels of snRNA-U6 and miR-661 in patients with nSS and autoantibody negative pSS patients are associated with normal $B$ cell activity and normal numbers of circulating leukocytes. Reduced miRNA levels in autoantibody positive pSS patients are associated with B cell hyperactivity and decreased leukocyte counts, which is possibly the result of immune cells migration to the inflammatory sites. Considering the important role of miRNAs in the control of immune cell activation, this work points to a significant role of miRNAs in pSS and nSS patients.

Disclosure of Interest: None declared

DOI: 10.1136/annrheumdis-2017-eular.4961

\section{AB0122 DETECTION OF AN INTRIGUING VIRUS-LIKE SEQUENCE IN THE SALIVARY GLAND EPITHELIAL CELLS OF SJÖGREN'S SYNDROME PATIENTS}

A.G. Vakrakou ${ }^{1,2}$, E. Karamichali ${ }^{3}$, O. Georgopoulou ${ }^{3}$, M.N. Manoussakis ${ }^{1,2}$. ${ }^{1}$ Pathophysiology, University of Athens; ${ }^{2}$ Laboratory of molecular immunology; ${ }^{3}$ Molecular Virology Laboratory, Hellenic Pasteur Institute, Athens, Greece

Background: Several previous studies from our laboratory had indicated that the salivary gland epithelial cells (SGEC) of primary Sjögren's syndrome (SS) patients manifest cell autonomous (intrinsic) activation, which is demonstrated in nonneoplastic cell lines derived from patients (SS-SGEC). As for other autoimmune diseases, it is widely proposed that infectious factors may incite SS. In fact, several viral agents have been proposed to be involved in the development of SS, including EBV, retroviruses (such as HTLV-1 and endogenous retrotransposons) and the Coxsackie virus. Recently, the sequence and antigen of HDV were detected in the salivary gland tissues of approximately half of SS patients studied [Weller et al., 2016], however, in the absence of detectable hepatitis B virus (HBV) surface antigen and antibodies to HBV or HDV. Therefore, we hypothesized that HDV may be causally related with the cell-autonomous aberrations observed in the SS-SGEC lines and may trigger the inflammatory and autoimmune reactions that characterize SS.

Objectives: Herein, using non-neoplastic SGEC lines derived from SS patients, we sought to address directly whether the SGEC of these patients manifest evidence for infection by the HDV virus, despite the fact that patients studied did not display any evidence of infection by HBV or HDV viruses.

Methods: For that purpose, we purified total RNA from SGEC lines from non-SS controls $(n=7)$ and SS patients $(n=7)$. Reverse transcription of RNA isolated from salivary gland epithelial cells (SGEC) was performed using random hexamer primers as per the manufacturer's specifications. We performed PCR for the identification of HDV RNA sequences with three sets of primers from different regions of the viral sequence. RNA extracted from HDV-positive sera was used as positive control in HDV-specific PCR.

Results: All SGEC lines from SS patients and non-SS controls tested were found positive for HDV RNA, as tested by the first two sets of primers (two sets of primers for first and nested PCR with expected PCR products $329 \mathrm{bp}$ and $234 \mathrm{bp}$, respectively). Nevertheless, when we selected primers for another region of the HDV genome (set of primers with expected PCR products $375 \mathrm{bp}$ ) all RNA samples from SGEC lines tested were found negative for HDV RNA, whereas the HDV RNA control specimen was positive.

Conclusions: We conclude that SS-SGEC are not actually infected by the HDV virus, but are probably carriers of an HDV-like sequence. In fact, the putative HDV virus-like sequence has been previously shown be highly homologous to the RNA binding-protein cytoplasmic polyadenylation element-binding protein-3 (CPEB3), which belongs to a family of genes regulating messenger RNA polyadenylation and is structurally and biochemically related to the human HDV ribozymes (Salehi-Ashtiani et al, 2006). We are currently in the process of further analyzing the nature of this HDV-like sequence, through cloning and Sanger sequencing of the RT-PCR products, as well as performing of HDV-specific immunostaining studies in both SGEC lines and salivary gland tissues.

Disclosure of Interest: None declared

DOI: 10.1136/annrheumdis-2017-eular.6308 